Progress in Flight Physics 5 (2013) 327-348

DOI: $10.1051 /$ eucass/201305327

C Owned by the authors, published by EDP Sciences, 2013

\title{
THREE-DIMENSIONAL SHOCK-WAVE/BOUNDARY-LAYER INTERACTION AT THE PRESENCE OF ENTROPY LAYER
}

\author{
V. Borovoy, I. Egorov, A. Maximenko, V. Mosharov, \\ V. Radchenko, A. Skuratov, and I. Struminskaya
}

Central Aerohydrodynamic Institute (TsAGI)

Zhukovsky, Moscow Region 140180, Russia

\begin{abstract}
An experimental and numerical investigation of a gas flow on a flat plate near a single fin and a fin pair, generating crossings shocks, is performed. The study is focused on the plate bluntness influence on the flow field and the heat transfer in the interaction region. The experiments are carried out in a short duration wind tunnel at Mach numbers $\mathrm{M}=5$, 6 , and 8 and Reynolds numbers $\operatorname{Re}_{\infty L}$ up to $27 \cdot 10^{6}$. Luminescent substances are used for heat flux and pressure distribution measurements and for the surface flow visualization. In addition, the heat flux is measured with thermocouple sensors. For a numerical flow simulation, the three-dimensional (3D) Reynolds-averaged Navier-Stokes (RANS) equations are solved using the $q-\omega$ turbulence model. It is found that even a small plate blunting affects heat transfer and pressure distributions significantly. Moreover, in the case of crossing shocks, it can cause a global transformation of the flow structure in the area of the interaction between the shock waves and the boundary layer.
\end{abstract}

\section{INTRODUCTION}

A great number of works (see, for example, $[1-8]$ ) are dedicated to the investigation of the interaction of the oblique shock waves, generated by a single fin or a fin pair with the boundary layer of the plate on which they are installed. First, experimental investigations were carried out. During the last two decades, high attention has been also paid to the numerical simulation of such flows. The review of the experimental and computational works performed up to 2003 is presented in [7].

Almost in all the works, the interference flow on a sharp plate was studied. At the same time, the investigations of two-dimensional (2D) interference between an oblique shock wave and the laminar boundary layer of the plate [9-11] show

This is an Open Access article distributed under the terms of the Creative Commons Attribution License 2.0, which permits unrestricted use, distribution, and reproduction in any medium, provided the original work is properly cited. 
that even a small bluntness of the plate leading edge significantly decreases the heat flux in the shock incidence region. It was also found that there is a threshold value of the plate blunting radius [10]: up to some treshold values, the maximum heat-transfer coefficient decreases significantly with an increase of the radius; once the treshold radius is reached, further blunting just weakly affects the maximum value of the heat-transfer coefficient. These peculiarities are related to the influence of the high-entropy layer generated by the blunted leading edge on the flow in the separation zone caused by the incident shock wave.

The influence of slight blunting on the gas flow and the heat transfer in the region of interaction between a single fin and the plate at Mach number $M=6$ and Reynolds numbers $\operatorname{Re}_{\infty L}$ up to $19.2 \cdot 10^{6}$ was investigated in [12]. The undisturbed boundary layer ahead of the fin was laminar or transitional. It was shown that with the boundary layer ahead of the fin being laminar, the maximum Stanton number behind the shock reaches approximately the same value as in the case of a turbulent flow or even exceeds this value. Slight blunting of the plate leading edge leads to a significant decrease of the maximum Stanton number and to some expansion of the region of the amplified heat flux. The heat transfer decrease caused by the plate blunting occurs only in the restricted range of the blunting radius $\left(r / X_{0} \leq 0.02-0.03\right)$. With $r / X_{0} \geq 0.03$, the blunting influence is negligible.

In the present work, the interaction of shock waves with the turbulent boundary layer at $M=5$ and 6 and laminar boundary layer at $M=8$ is investigated. The flow in the vicinity of a single fin and a fin pair, generating crossing shocks, is studied. In the second case, the contraction ratio of the channel between the fins is varied.

\section{MODELS. FLOW CHARACTERISTICS}

The main part of the models is the plate 1 (Fig. 1). Three variants of the plates are used:

(1) a steel plate destined for measuring the heat transfer coefficient by the "thin wall" sensors. The sensors are placed in three sections (see Fig. 1): at the symmetry line and in two cross sections;

(2) a similar steel plate with a thermoinsulating coating made of glassreinforced plastic. It is designated for heat transfer measurements using temperature sensitive paint (TSP); and

(3) a plate made of an aluminum alloy. It is designated for pressure distribution measuring using pressure sensitive paint (PSP) and for the surface flow visualization by the oil, containing solid fluorescent particles. 


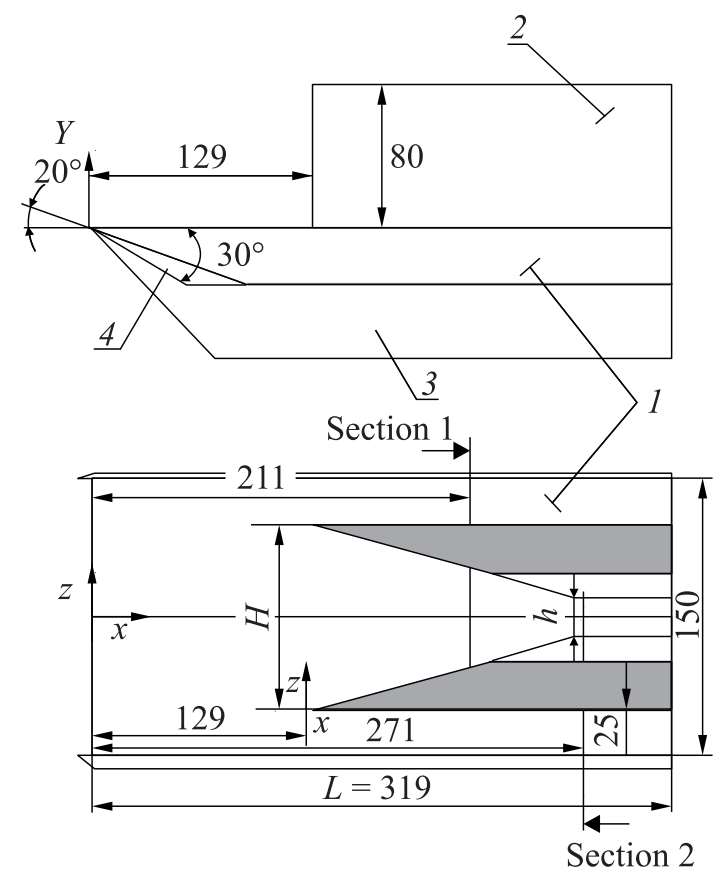

Figure 1 Model configuration and coordinate system. Dimensions are in millimeters

One fin or two asymmetric fins 2 are installed on the plate at a distance of $129 \mathrm{~mm}$ from its leading edge. All the fins have sharp leading edges. Single fins with flow deflection angles $\theta=10^{\circ}, 15^{\circ}$, and $20^{\circ}$ are tested. In the case of the fin pair, only the fins with $\theta=15^{\circ}$ are used. The fins of different thicknesses are used so that the contraction ratio of the channel between the fins is 2 or 4 . Replaceable front pads 4 are fixed on the plate to vary its bluntness. Additionally, the longitudinal fences 3 are installed on the plate sides to reduce the gas overflow from one plate surface to the other. The experiments are carried out in the TsAGI's shock wind tunnel UT-1M. It operates on Ludwieg

Table 1 Investigated flow characteristics

\begin{tabular}{cccc}
\hline $\begin{array}{c}\text { Mach number } \\
\mathrm{M}\end{array}$ & $\begin{array}{c}\text { Total pressure } \\
P_{t}, \text { bar }\end{array}$ & $\begin{array}{c}\text { Total temperature } \\
T_{t}, \mathrm{~K}\end{array}$ & $\begin{array}{c}\text { Reynolds number } \\
\operatorname{Re}_{\infty L}(L=0.32 \mathrm{~m})\end{array}$ \\
\hline 5 & 69 & 530 & $27 \cdot 10^{6}$ \\
6 & 90 & 560 & $19 \cdot 10^{6}$ \\
8 & 90 & 690 & $7 \cdot 10^{6}$ \\
\hline
\end{tabular}


tube scheme. Duration of the steady flow is $40 \mathrm{~ms}$. The characteristics of the undisturbed flow are presented in Table 1.

A natural laminar/turbulent boundary layer transition takes place on the plate ahead of the fins at Mach number $M=5$. At $M=6$, the trips are used for boundary layer turbulization near the plate leading edge. At $M=8$, the undisturbed boundary layer is laminar.

\section{INVESTIGATION METHODS}

The distributions of the heat transfer and of the pressure are investigated using luminescent TSP and PSP, respectively. The measurement methods are described in details earlier $[12,13]$. In the present work, the technology of TSP and PSP application is modified to provide the observation of the entire plate surface. Discrete measurements of the heat flux using the "thin wall" sensors are made also.

The visualization of the surface flow and the skin friction are made using the method recently developed in TsAGI [13]. Small displacements of the luminescent particles introduced in the oil are measured using this method.

For a numerical flow simulation, the 3D RANS equations are solved, using the $q-\omega$ turbulence model $[11,14]$.

\section{SINGLE FIN ON THE PLATE}

\subsection{Flow Pattern}

At a sufficiently high intensity of the shock generated by the fin, the boundary layer separates from the plate surface. The separation zone can be divided into two regions $[2,4]$ : the initial region located near the fin leading edge and the region of a developed separation distant from the fin leading edge. The length of the initial region is about $(10-12) \delta$ for the laminar undisturbed boundary layer ( $\delta$ is the layer's thickness at the fin leading edge), and it is equal to $(6-8) \delta$ for the turbulent one [2]. In the initial region, the flow characteristics vary significantly both along the fin and in the perpendicular direction. In the second region, the flow parameters vary slightly along the rays, originating from the virtual pole, situated in front of the fin. This region is referred to as quasi-conical region $[3,8]$. The majority of published works focus upon the second region. However, the length of the first region can constitute a significant part of the fin length or even embraces the fin completely. The length of the initial region increases at the displacement of the fin from the plate leading edge (with boundary-layer thickening). This investigation shows 


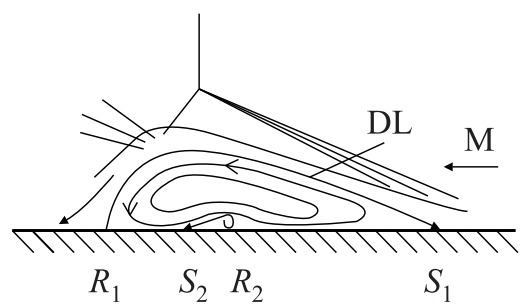

(a)

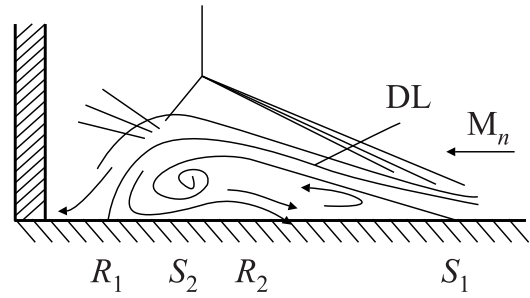

(b)

Figure 2 Flow patterns: 2D (a), and 3D (b) separation flows

that increase of the plate bluntness and Mach number also elongates the first region.

There is an essential difference in the flow patterns of 2D and 3D separation flows. The first case takes place when the separation line is normal to the undisturbed flow direction. The second case corresponds to the flow near a fin. At the 2D separation flow, a closed separation zone is formed (Fig. 2a) [3]. Dividing streamline separated from the plate surface at the point $S_{1}$ and reattached to it at the point $R_{1}$, is formed. In the vicinity of the point $R_{1}$ the heat flux and the pressure increase dramatically.

At the $3 \mathrm{D}$ separation flow near a sharp fin, the separation zone expands with the distance from the fin leading edge. It leads to an increase of the flow rate inside the separation zone. Therefore, the gas inflows into the separation zone continuously, and the boundary of the separation zone cannot be closed (Fig. 2b): the streamline separated at the point $S_{1}$ does not reattach to the surface; instead, it moves downstream in a spiral form, and another streamline DL displaced from the plate surface (on the distance $Y_{s}$ ) attaches to the plate. The necessary additional gas mass inflows into the separation area between the plate surface and the dividing line DL. Such separation flow pattern near a fin is confirmed by the numerical flow simulation in [4].

In the initial region, the attaching flow jets are characterized by low values of the total pressure $P_{t}$ and the stagnation temperature $T_{t}$. First, this is because they emanated at the small distance $Y_{s}$ from the plate surface. Second, due to a small lateral dimension of the separation zone, the separated jet cannot increase the values of $P_{t}$ and $T_{t}$ significantly. The heat transfer and pressure coefficients on the reattachment line increase gradually with the distance from the fin leading edge.

At a far distance from the fin leading edge (in the region of the quasi-conical flow), where reattaching jets are originating in the external part of the boundary layer (or outside the boundary layer), the parameters $P_{t}$ and $T_{t}$ of reattaching jet are approaching to their values in the external flow (in the case of a sharp 
plate) or in the high-entropy layer (in the case of a blunted plate). Farther, the parameters $P_{t}$ and $T_{t}$ are varying with the distance from the fin leading edge weakly.

Even slight blunting of the plate affects the flow and the heat transfer near the reattachment line significantly. The blunted leading edge generates a highentropy layer. Similar to the boundary layer, some part of the high-entropy layer cannot penetrate without separation into the region, situated behind the strong shock, and is involved into the circulation together with the boundary layer. As a result, the bluntness causes a thickening of the mixing layer and, therefore, an increase of all dimensions of the separation zone generated by the shock. The thickening of the mixing layer, as well as the decrease of the gas density in it, leads to the decrease of the heat-transfer coefficient in the vicinity of the reattachment line. This follows from the fact that the mixing-layer thickness is the only characteristic dimension in the reattachment region.

Only a restricted amount of gas contained in the high-entropy layer can be sucked by the mixing layer. Therefore, the thickening of the mixing layer due to the increase of the bluntness radius takes place only up to a certain bluntness value. In addition, after the high-entropy layer achieves the reattachment point $R$, the gas density and other characteristics on the external boundary of the mixing layer correspond to the characteristics of the high-entropy layer and do not depend on its thickness. As a result, starting from some threshold radius, the subsequent increase of bluntness radius does not affect the heat transfer in the interference region significantly.

The experimental and computational data presented below specify the flow characteristics in the region of interaction between a fin and a fin pair with the plate.

\subsection{Distributions of Heat-Transfer and Pressure Coefficients in the Interference Zone}

The distributions of the Stanton number St, the pressure coefficient $C_{p}$, and surface streamlines on the surface of $\operatorname{sharp}(r=0)$ and blunted $(r=0.75 \mathrm{~mm})$ plates with the fin $\theta=15^{\circ}$ are presented in Fig. 3. It is evident that the separation zone is formed near the fin. The Stanton number, the surface pressure, and the skin friction (the skin friction is displayed as a false-color distribution) sharply increase on the reattachment line (near the fin). The comparison of data for the sharp and blunted plates shows that the plate blunting leads to the reduction of the heat transfer, the pressure, and the friction. Simultaneously, the region of the fin influence is expanded.

Figure 3 also illustrates that the heat transfer and pressure coefficients are increased gradually with the distancing from the fin leading edge along the reattachment line. Figure 4 presents this fact quantitatively. The tendency of the 


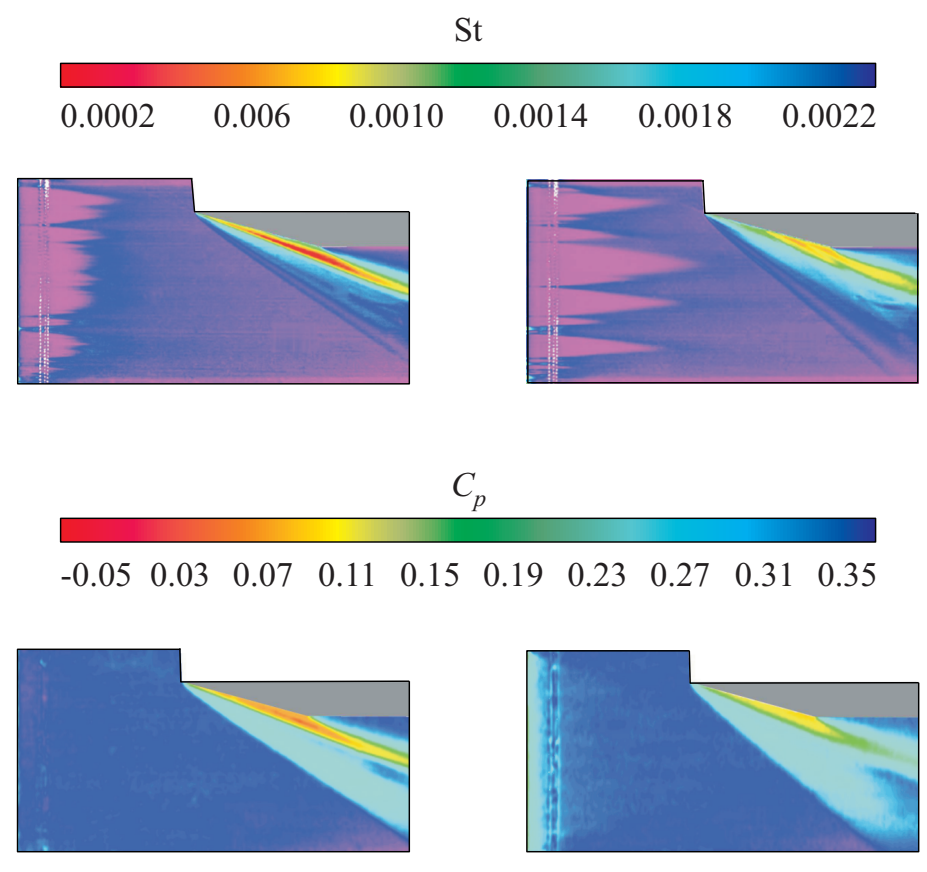

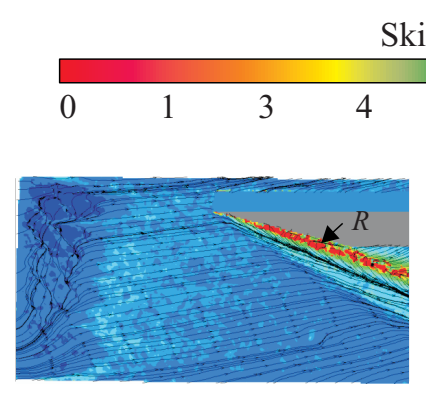

(a)
Skin friction

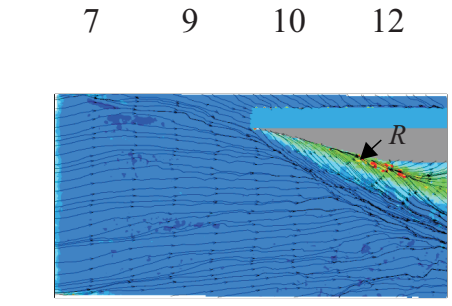

(b)

Figure 3 Flow fields on the sharp and blunted plates at $\mathrm{M}=5, \operatorname{Re}_{\infty L}=27 \cdot 10^{6}$, and $\theta=15^{\circ}:(a)$ sharp plate $(r=0)$; and $(b)$ blunted plate $\left(r=0.75 \mathrm{~mm}, r / X_{0}=5.8 \cdot 10^{-3}\right.$, $X_{0}$ is the distance of fin leading edge from the plate leading edge), $R$ indicates the reattachment line. 

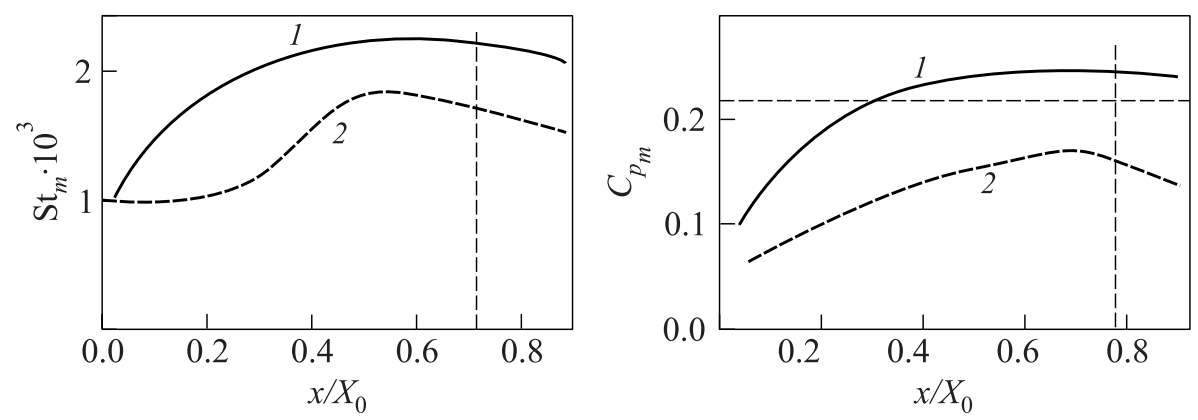

Figure 4 Longitudinal distributions of Stanton number and pressure coefficient on the reattachment line at $\mathrm{M}=5$ and $\theta=15^{\circ}$ : vertical dashed line - angular point on the fin; horizontal dashed line $-C_{p}$, calculated for the nonviscous flow; $1-r=0 \mathrm{~mm}$; and $2-r=0.75 \mathrm{~mm}$
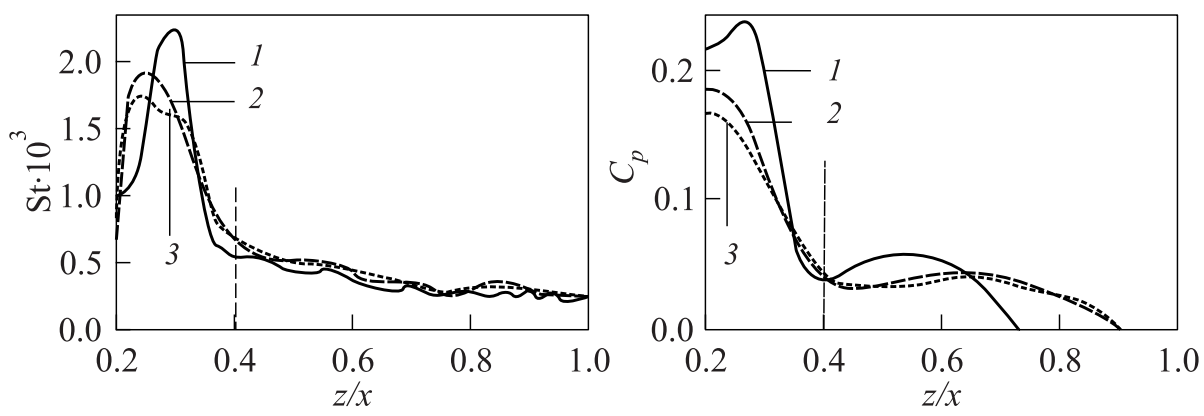

Figure 5 Lateral distributions of Stanton number and pressure coefficient in the cross section $x / X_{0}=0.64$ at $\mathrm{M}=5$ and $\theta=15^{\circ}: 1-r=0 \mathrm{~mm} ; 2-0.5$; and $3-$ $r=0.75 \mathrm{~mm}$

initial zone to elongate at the reduction of the fin angle $\theta$ is observed: at $\theta=10^{\circ}$ the initial zone is significantly longer than at $\theta=15^{\circ}$. The plate leading edge blunting also elongates the initial region of interference.

For example, at $\theta=10^{\circ}$, the increase of plate bluntness radius from $r=0$ to $0.75 \mathrm{~mm}$ almost doubles the length of the initial region. At $\theta=20^{\circ}$, the initial region of interference occupies the entire length of the fin (up to the angular point) even on the sharp plate (however, the fin length at $\theta=20^{\circ}$ is shorter than at $\theta=10^{\circ}$ and $15^{\circ}$, because all the fins used in optical measurements have the same thickness $b=25 \mathrm{~mm}$ ).

Figure 5 presents the lateral distributions of the heat transfer and pressure coefficients near the fin $\theta=15^{\circ}$ in the cross section $x / X_{0}=0.64$, which is 
situated in the region of the quasi-conical flow. It can be seen that two maxima of pressure are formed: the absolute maximum is located behind the shock wave on the line of primary reattachment, and the local maximum is located on the reattachment line behind the secondary separation. In the Stanton number distribution, the absolute maximum of the Stanton number can be seen also, but the local maximum is undistinguished here. This can be explained by the insufficient distance between the section $x / X_{0}=0.64$ and the fin leading edge. Both the local minimum and the local maximum of the heat flux can be seen in Fig. 3 at a larger distance from the fin leading edge (behind the angular point).

\subsection{Influence of the Plate Bluntness on the Maximum Values of Heat Transfer and Pressure Coefficients}

The maximum values of Stanton number $\mathrm{St}_{m}$ and the pressure coefficient $C_{p_{m}}$ in the section $x / X_{0}=0.64$ at different fin angles are presented in Fig. 6 as functions of $\xi$ where $\xi$ is the pressure ratio behind the shock $P_{2}$ and in front of it $P_{1}$ at the nonviscous flow $\left(\xi=P_{2} / P_{1}\right)$. The value of $\mathrm{St}_{m}$ is normalized by the turbulent value of Stanton number calculated for the sharp plate in the same section. The heat transfer coefficient is measured by the "thin wall" sensors near the thick fins $(b=37.5 \mathrm{~mm})$; and the surface pressure is measured by PSP near the fins of $25 \mathrm{~mm}$ thickness. In the case of a sharp plate, the presented results are in agreement with data of Schulein [8]. The discrepancies are about $15 \%$. They are caused by:
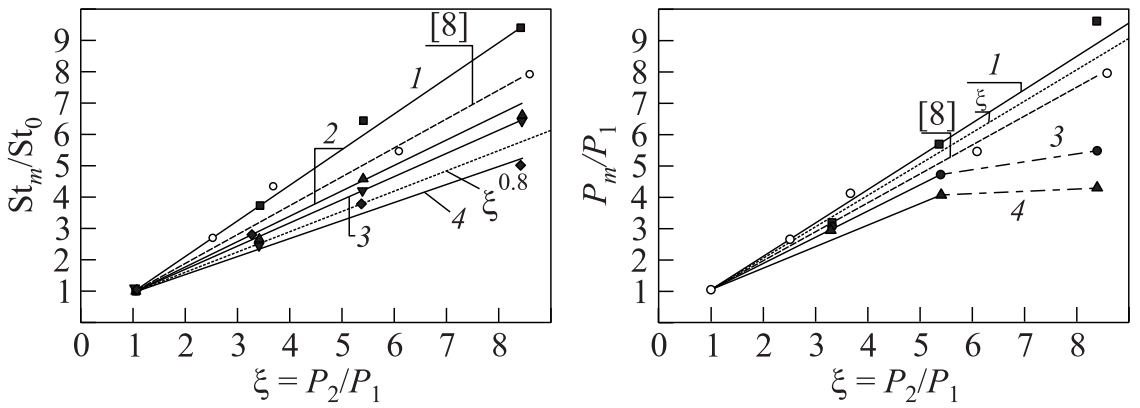

Figure 6 Maximum heat transfer amplification $\mathrm{St}_{m} / \mathrm{St}_{0}$ and maximum pressure increase $P_{m} / P_{1}$ in cross section $1\left(x / X_{0}=0.64\right)$ at $\mathrm{M}=5$ and $\operatorname{Re}_{\infty L}=27 \cdot 10^{6}$ vs. the calculated pressure ratio $\xi$ in the oblique shock wave: $1-r=0 \mathrm{~mm} ; 2-0.5 ; 3-$ 0.75 ; and $4-r=2 \mathrm{~mm}$ 


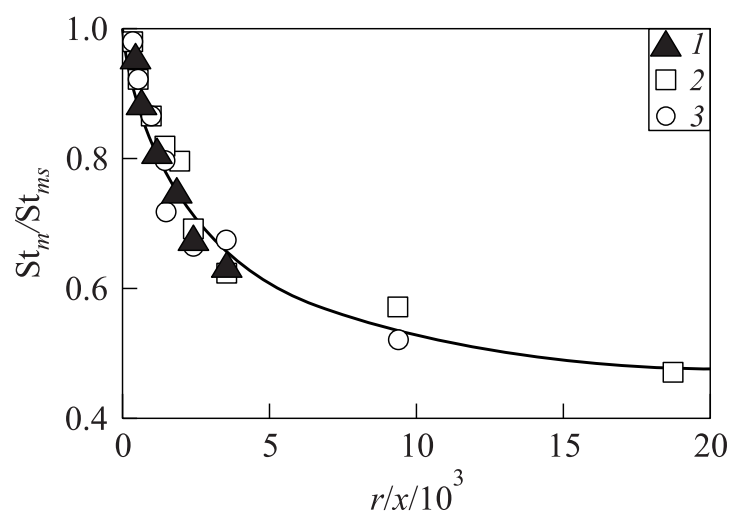

Figure 7 Maximum Stanton number in cross section $1\left(x / X_{0}=0.64\right)$ at $\mathrm{M}=5$ vs. the relative bluntness radius: $1-\theta=10^{\circ} ; 2-15^{\circ}$; and $3-\theta=20^{\circ}$

(i) errors of measurements;

(ii) some differences in the flow conditions (in [8] the measurements were carried out at $\left.x / X_{0} \geq 0.3\right)$; and

(iii) different determination of undisturbed values (in [8], $\mathrm{St}_{0}$ and $P_{1}$ were determined experimentally).

Figure 7 demonstrates the influence of the plate bluntness on the ratio $\mathrm{St}_{m} / \mathrm{St}_{m s}$. St $\mathrm{St}_{m s}$ is the maximum value of the Stanton number on the sharp plate in the same section $x / X_{0}=0.64$. It can be seen that at all the investigated fin angles, the plate blunting reduces the heat transfer near the fin in an equal degree. A significant reduction of the heat transfer occurs at the increase of the plate bluntness radius only up to $r / X_{0} \approx 0.010-0.015$, i. e., at the turbulent state of the undisturbed boundary layer, the stabilization of the ratio $\mathrm{St}_{m} / \mathrm{St}_{m s}$ occurs at smaller relative bluntness radius than at the laminar and transient states of the boundary layer $(0.02-0.03)[12,13]$.

\subsection{Comparison of Computational Results with Experimental Data}

The calculated heat transfer distributions at $\mathrm{M}=5$ on the plate near the fin with the angle $\theta=15^{\circ}$ are compared with the experimental data in Fig. 8. The measurements were made by both the thermocouple sensors and the TSP. The computational fluid dynamics (CFD) results comply satisfactorily with the experimental data, and computational results are closer to the experimental data obtained by the thermocouple sensors which are more accurate than the results of optical measurements. 


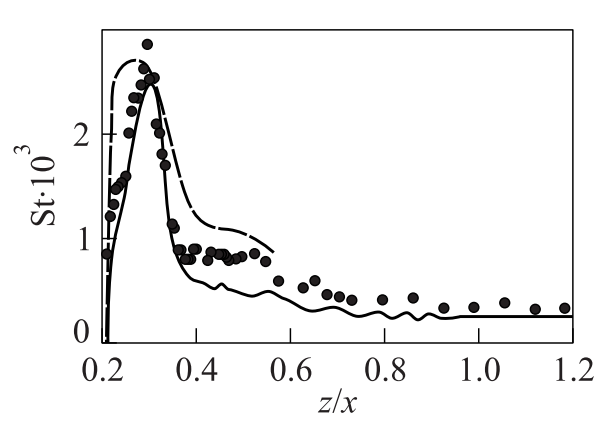

(a)

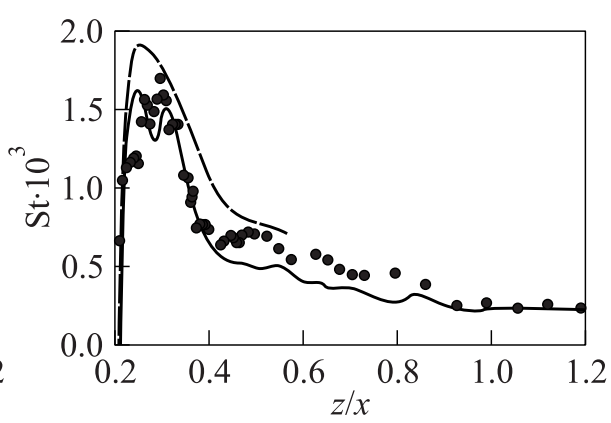

(b)

Figure 8 Stanton number distribution in cross section $1\left(X=211 \mathrm{~mm}, x / X_{0}=0.64\right)$ at $\mathrm{M}=5, \theta=15^{\circ}$; dashed lines - CFD, solid lines — TSP, signs - thermocouples: (a) $r=0 \mathrm{~mm}$; and (b) $r=0.75 \mathrm{~mm}$

\section{FIN PAIR ON THE PLATE}

\subsection{Flow Patterns}

The flow structures near the fins, generating crossing shocks, are displayed in Figs. 9 and 10. Figure 9 corresponds to the sharp plate. This case is investigated in details experimentally and numerically $[7,8]$. The main shock waves 1 , generated by the fins, cause boundary layer separation. The boundary layer separates on the lines 2 and reattaches near the fins, on the lines 3 . The separation zones in turn generate the separation shocks. In contrast to the main shocks, the sep-
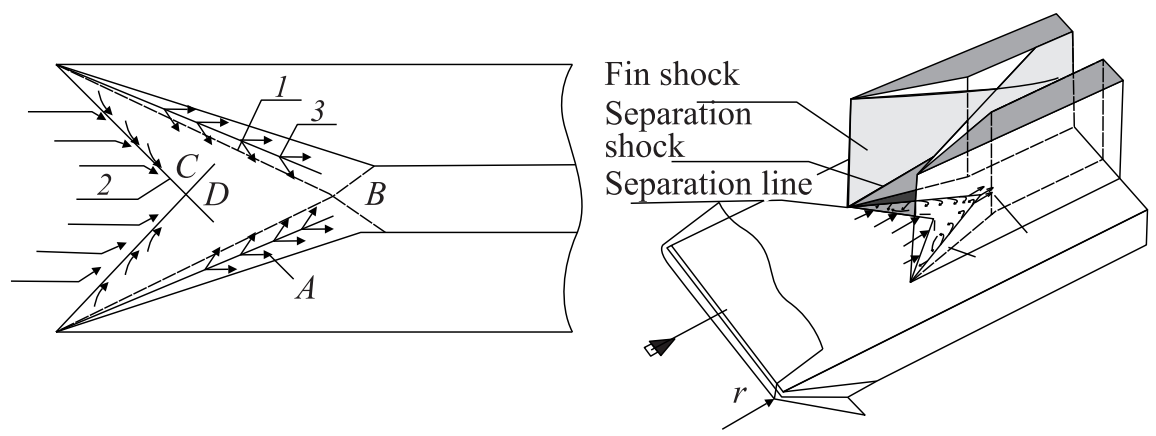

Figure 9 Regular flow structure near a fin pair installed on a sharp or slightly blunted plate: 1 - fin shocks; 2 - separation lines; 3 - reattachment lines; and $A, B, C$, and $D$ - areas of enhanced heat transfer 

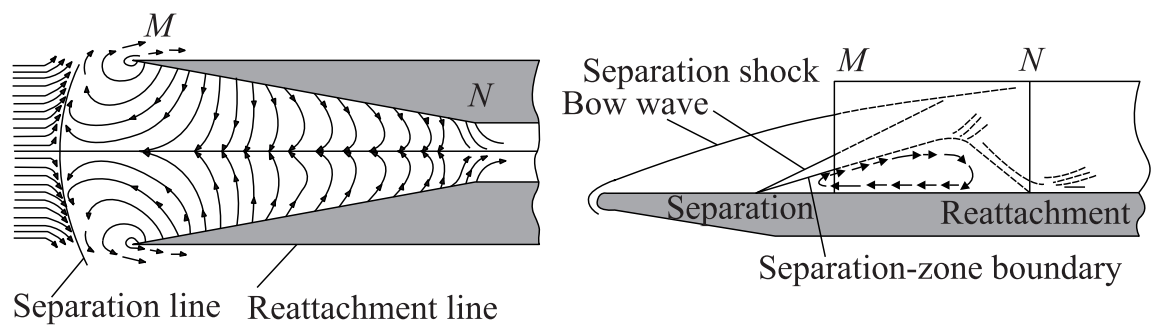

Figure 10 Nonregular flow structure near a highly-blunted plate

aration shocks are inclined not only to the undisturbed flow direction but also to the plate surface. The regions of amplified heat transfer and high pressure (regions $A$ ) are formed behind the shocks 1 (in the vicinity of the divergence lines 3 ), as in the case of a single fin. A region of even higher pressure and more intensive heat transfer (region $B$ ) is formed behind the intersection point of the main shocks. Behind the separation shocks (in the regions $C$ ), some increase of the heat transfer and pressure takes place. Finally, one more region of intensive heat transfer (region $D$ ) is formed behind the intersection point of the separation shocks. The lines of secondary separation, which are not shown in Fig. 9, are generated inside each zone of separation.

The blunted leading edge of the plate generates an entropy layer. A part of this layer is involved into the separation flow near the fins. Due to the increase of mass, involved into the separation flow, the separation zones are expanding and the separation lines 2 are displacing forward, towards the undisturbed flow. Correspondingly, the oblique separation shocks are displacing forward, and the angle between them and the undisturbed flow direction is increasing. However, at small bluntness, the regular flow with the plane separation shocks shown in Fig. 9 is kept.

At a certain bluntness value, a principle change of the flow occurs at a high contraction ratio: two plane separation shocks are merged in one curved separation shock, it is expelled forward and its origin is located in front of the fins (see Fig. 10). The boundary layer also separates in front of the fins and reattaches partly near the fins (as in the case of the regular flow) and partly at the throat of the channel, formed between the fins. From the throat, one part of the separated gas moves back and the other one continue flowing in the normal direction.

The expected reason of the flow transformation consists in the following: at a sufficiently high inclination angle of the separation shocks (with respect to the undisturbed flow), the regular interaction of the separation shocks changes into the Mach interaction; it leads to the formation of strong shock and to the generation of a jet with a reduced total pressure. At a small throat width, this 
results in chocking of the near-wall part of the channel and to the expelling of the separation shocks.

The picture observed is similar to the unstart of a convergent channel. There are significant differences however: in the case of the unstart, the flow behind the expelled bow wave becomes subsonic, with a high loss of the total pressure. In the considered case, the flow outside the separation zone even at the expelled separation shocks remains supersonic, and the total pressure losses are not so high as in the case of the unstart. In the case of unstart the entire flow behind the channel leading edges transforms, while in the considered case, only the near-wall part of the channel flow varies significantly. However, the bluntness influence can be more significant if the fins are situated between two plates: the blunting of the plates can cause unstart of the channel even at a relatively small contraction of the channel.

The flow structures presented in Figs. 9 and 10 are based on the data provided below. The fields of Stanton number St and pressure coefficient $C_{p}$ are displayed in Figs. 11 and 12 for the sharp and blunted plates at $M=5$ and $\operatorname{Re}_{\infty L}=27 \cdot 10^{6}$
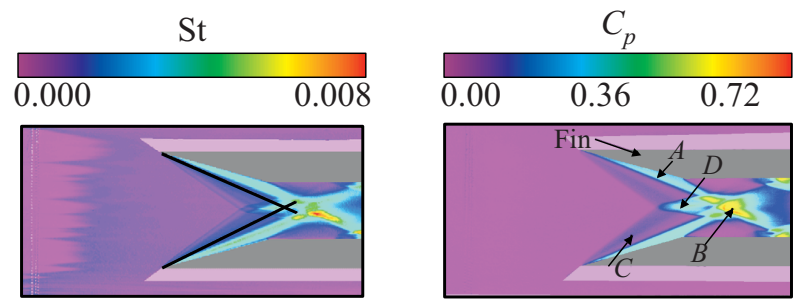

(a)
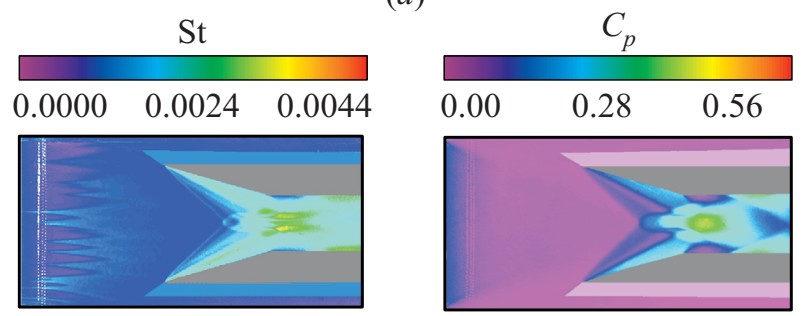

(b)

Figure 11 Flow fields near the fin pair at $\mathrm{M}=5, \operatorname{Re}_{\infty L}=27 \cdot 10^{6}$, and channel contraction ratio $H / h=2:(a) r=0 \mathrm{~mm}$; and $(b) r=2 \mathrm{~mm}$. Left column refers to Stanton numbers and right column refers to pressure coefficient. 

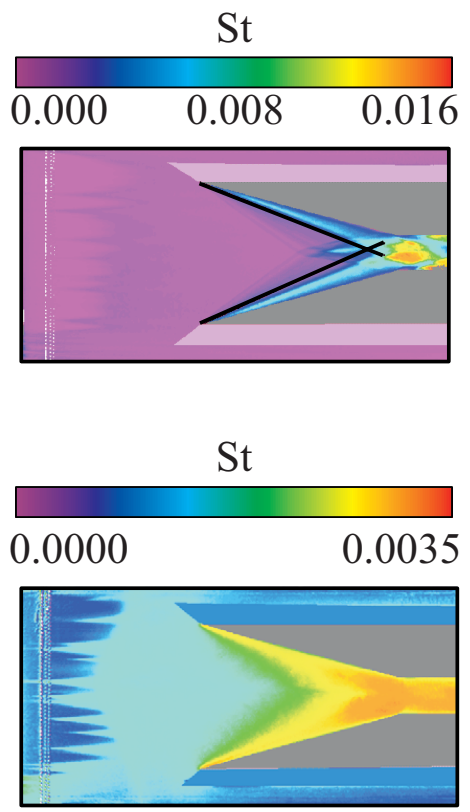

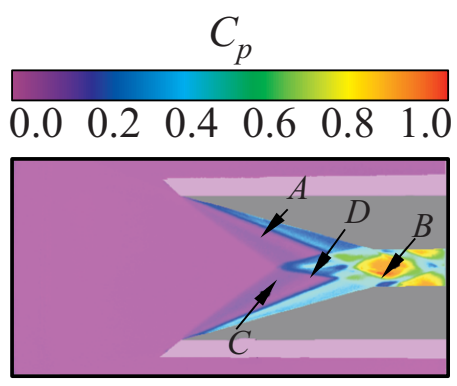

(a)
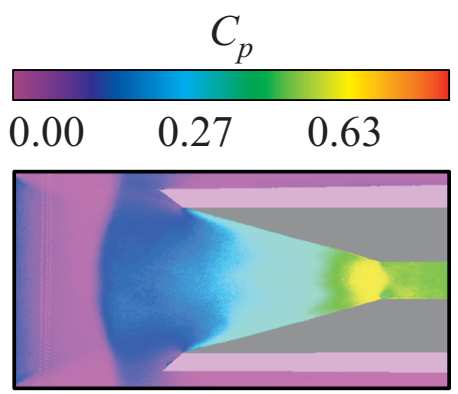

(b)

Figure 12 Flow fields near the fin pair at $\mathrm{M}=5, \operatorname{Re}_{\infty L}=27 \cdot 10^{6}$ and channel contraction ratio $H / h=4:(a) r=0 \mathrm{~mm}$; and $(b) r=2 \mathrm{~mm}$. Left column refers to Stanton numbers and right column refers to pressure coefficient.

in two cases: at the channel contraction ratio $H / h=2$ and $4(H=100 \mathrm{~mm})$. In addition, the surface flow visualization and the schlieren photos for the case $H / h=4$ are presented in Fig. 13 .

At a small channel contraction ratio (at $H / h=2$, see Fig. 11), the regular flow is observed on the sharp plate. Mach interference between the separation shocks can be supposed at the bluntness $r=2 \mathrm{~mm}$. But it does not result in shocks expelling at the contraction ratio $H / h=2$. The plate blunting reduces the heat transfer and the pressure in the interference region. Simultaneously, it leads to expanding of regions of the amplified heat transfer and the increased pressure.

In the case of high channel contraction ratio (at $H / h=4$, see Fig. 12 ), the behavior of the heat transfer coefficient distribution sharply changes at a certain value of the plate bluntness radius (in the considered case, at $r=2 \mathrm{~mm}$ ). It is observed in Figs. 12 and 13 that at $r=2 \mathrm{~mm}$, the separation line is formed in front of the fins. Behind it, the heat transfer and pressure coefficients increase, 


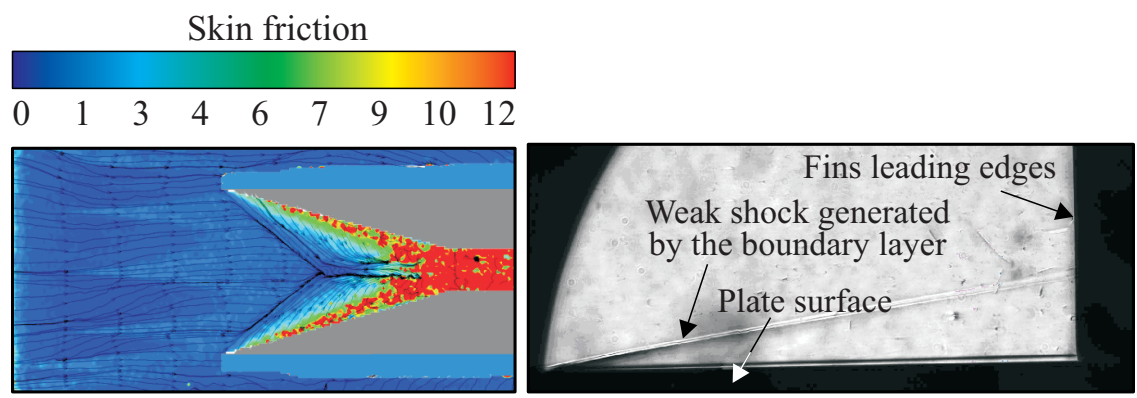

(a)
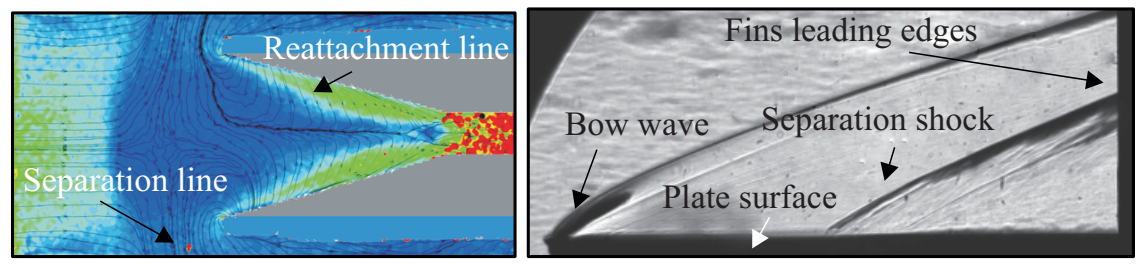

(b)

Figure 13 Surface streamlines, skin friction, and schlieren photos at $M=5, \operatorname{Re}_{\infty L}$ $=27 \cdot 10^{6}$, and channel contraction ratio $H / h=4:(a) r=0.5 \mathrm{~mm}$; and $(b) r=2 \mathrm{~mm}$.

and the skin friction decreases. Skin friction remains at a high level only behind the reattachment line (behind the channel throat). At the flow with expelled separation shocks, only one region of amplified heat transfer is observed, namely the region $B$. It is caused by the formation of the divergence line and the pressure peak at the end of separation zone. At $r=0.5 \mathrm{~mm}$, only the bow wave generated by the blunted leading edge is observed on the shadow photo in Fig. 13, because the main oblique shocks and the separation shocks are "hidden" by the fins. At $r=2 \mathrm{~mm}$, it is possible to see in Fig. 13 the separation shock, too. Its inclination angle (about $30^{\circ}$ ) corresponds to the high inclination angle of the separation zone boundary (about $20^{\circ}$ at $\mathrm{M}=5$ ) that is typical for the shock/turbulent-boundary-layer interaction.

A few experiments have been carried out at the same Mach number $\mathrm{M}=5$ but at a reduced total pressure $\left(P_{t}=14\right.$ bar and $\left.\operatorname{Re}_{\infty L}=5.8 \cdot 10^{6}\right)$. In this case, the separation shocks are expelled even at the plate bluntness radius $r=0.75 \mathrm{~mm}(H / h=4)$. The inclination angle of the separation shock (about $20^{\circ}$ ) corresponds to the relatively small inclination angle of the separation zone boundary $\left(10^{\circ}\right)$, as in the case of laminar flow. The higher influence of the plate blunting at the reduced total pressure is connected with thicken- 
ing of the boundary layer and the corresponding expansion of the separation zones.

\subsection{Distribution of Heat Transfer and Pressure Coefficients in the Interference Zone}

The distribution of the Stanton number St (thermocouple sensors data) and the pressure coefficient $C_{p}$ (PSP data) in section 1 situated in front of the channel throat is presented in Fig. 14. The coordinate system shown in Fig. 1 is applied (the origin is situated near the fin leading edge). In the considered section, the fins interaction is noticeable only near the symmetry line, whose position is characterized by the ratio $z / x=0.61$. In the remaining part of section 1 , the distributions of St and $C_{p}$ at both contraction ratios $H / h=2$ and 4 practically coincide within the range of the blunting radii from $r=0$ to $r=0.5 \mathrm{~mm}$ (only the data for $r=0$ are presented in Fig. 14 at $H / h=2$ ). Figure 14 demonstrates the possibility of two flow structures at $r=0.75 \mathrm{~mm}$ : the regular flow (at the measurements of the heat transfer coefficient, Fig. 14a) and the flow with expelled separation shocks (at the pressure measurements, Fig. 14b). The observed instability of the flow structure can be related both to some uncertainty of the test regime and to the difference in the surface roughness and in its temperature. At the regular flow, the plate blunting reduces the heat transfer and decreases the degree of the pressure rise.

The Stanton number distributions in section 2 at high and small channel contraction ratios are presented in Fig. 15. Here, the lateral coordinate $z$ is normalized by the channel width $h$. Unfortunately, the fins where installed at some angle relatively to undisturbed flow direction (at about $50^{\prime}$ ). Therefore,

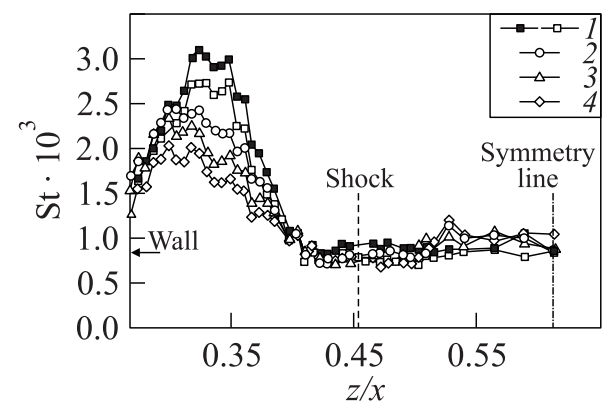

(a)

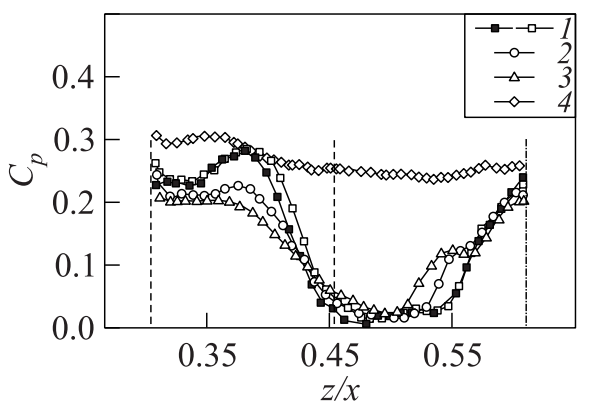

(b)

Figure 14 Stanton number $(a)$ and pressure coefficient $(b)$ distributions in cross section $1\left(x / X_{0}=0.64\right)$ at $\mathrm{M}=5: 1-r=0 \mathrm{~mm} ; 2-0.3 ; 3-0.5 ;$ and $4-$ $r=0.75 \mathrm{~mm}$. Empty symbols refer to $H / h=4$ and filled symbols to $H / h=2$ 


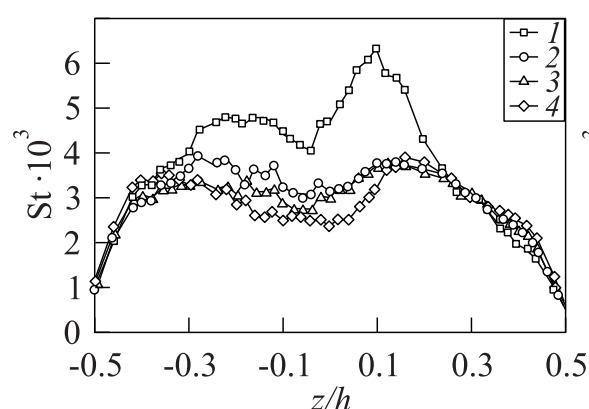

(a)

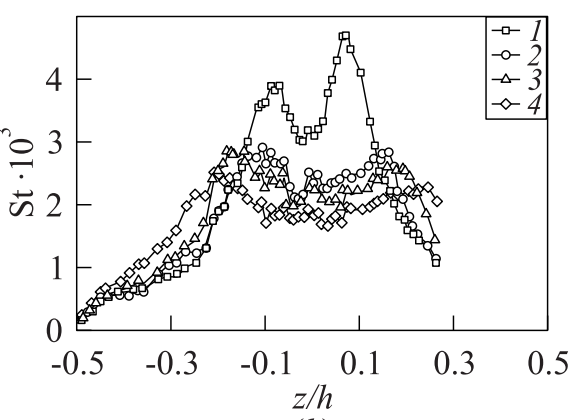

(b)

Figure 15 Stanton number distributions between the fins in cross section $2\left(x / X_{0}\right.$ $=1.1)$ at big and small channel contraction ratios $\left(\mathrm{M}=5, \operatorname{Re}_{\infty L}=27 \cdot 10^{6}\right):(a) H / h$ $=4 ;(b) H / h=2 ; 1-r=0 \mathrm{~mm} ; 2-0.3 ; 3-0.5$; and $4-r=0.75 \mathrm{~mm}$

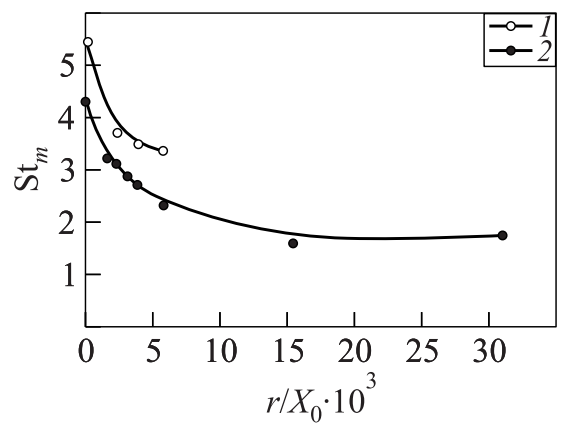

(a)

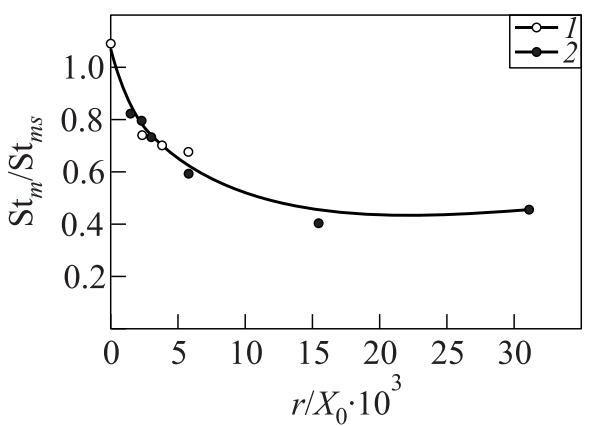

(b)

Figure 16 Maximum Stanton number in cross section 2 vs. the relative plate blunt-

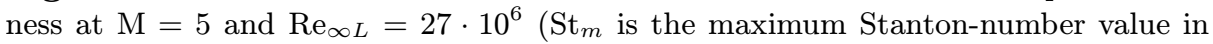
the cross section; and $\mathrm{St}_{m s}$ is the same for the sharp plate): $1-H / h=4$; and $2-$ $H / h=2$

the asymmetry of the heat transfer distribution is observed. As the blunting radius increases, the heat transfer intensity in section 2 significantly reduces, as in section 1. Simultaneously, the asymmetry of the Stanton number distribution decreases.

The maximum values of the Stanton number $\left(\mathrm{St}_{m}\right)$ and pressure coefficients $\left(C_{p_{m}}\right)$ in section 1 depend slightly on the channel contraction ratio. But in section 2 located behind the channel throat, $\mathrm{St}_{m}$ is approximately 1.5 times higher at $H / h=4$ than at $H / h=2$ due to the higher gas compression (Fig. 16a). At the same time, the ratio $\mathrm{St}_{m} / \mathrm{St}_{m s}$ in both sections depends, mainly, only on 
the normalized blunting of the plate leading edge: an increase of $r / X_{0}$ from 0 to $\approx 0.015$ causes the decrease of Stanton number $\mathrm{St}_{m}$ by $50 \%-60 \%$, and the further increase of $r / X_{0}$ influences the value of $\mathrm{St}_{m}$ lightly (Fig. 16b).

\subsection{Influence of Mach Number on the Flow and Heat Transfer}

The results obtained at $\mathrm{M}=6$ are similar to the above presented results for $\mathrm{M}=5$. But at $\mathrm{M}=6$, the flow transformation occurs at a lower blunting $(r=0.75 \mathrm{~mm})$ than at $\mathrm{M}=5(r=2 \mathrm{~mm})$ (both investigations are performed on the same model during heat transfer measurements).

The test results, obtained at $\mathrm{M}=8$ and $\operatorname{Re}_{\infty L}=7 \cdot 10^{6}$, are presented in Figs. $17-19$. At a high channel contraction ratio (at $H / h=4)$, even the small plate blunting of $0.3 \mathrm{~mm}$ causes the flow transformation (Fig. 17). The inclination angle of the separation shock $\left(12.7^{\circ}\right)$ corresponds to the small inclination angle of the separation zone boundary $\left(7^{\circ}\right)$, characteristic for the laminar flow.

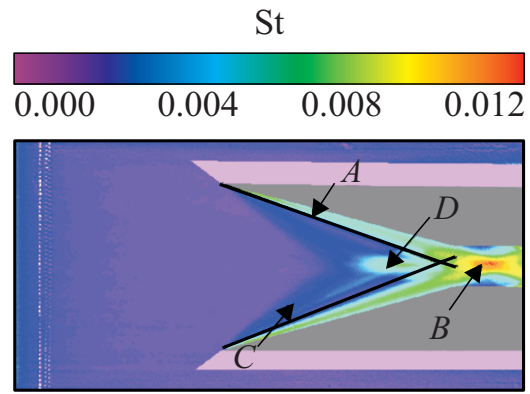

St

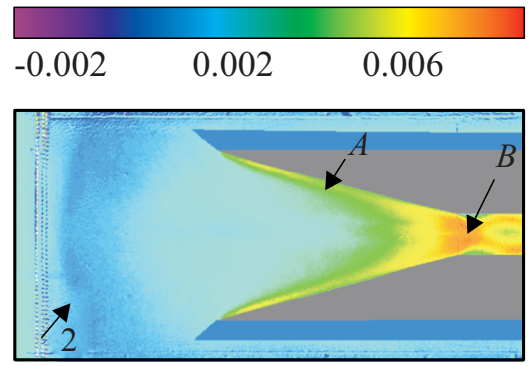

\footnotetext{
$(b)$
}

Fins leading edges

Weak shock generated by the boundary layer

\section{Plate surface}

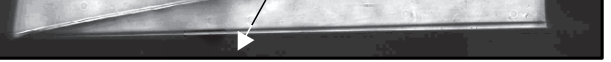

(a)

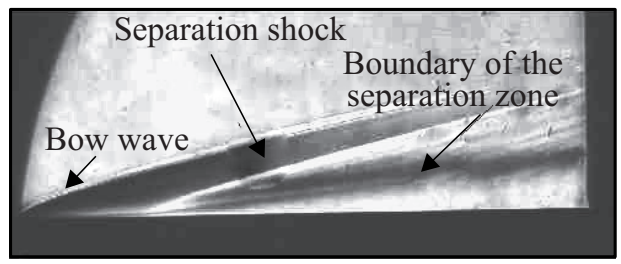

Figure 17 Flow fields at $\mathrm{M}=8$ and $H / h=4:(a) r=0 \mathrm{~mm}$; and $(b) r=0.3 \mathrm{~mm}$. Left column refers to Stanton number and right column refers to schlieren photos. 


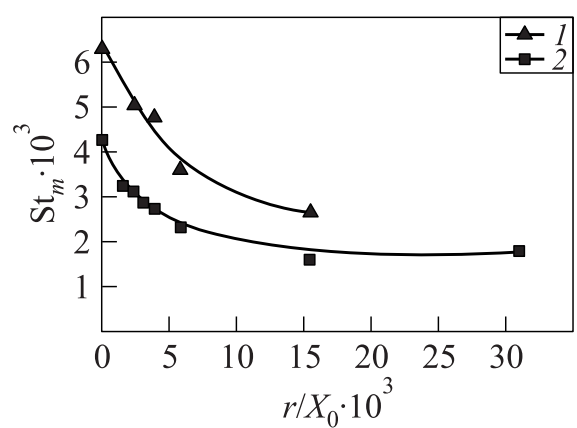

(a)

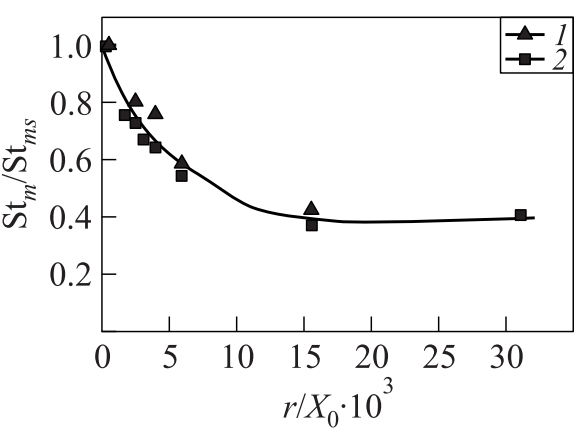

(b)

Figure 18 Maximum Stanton number in cross section 2 vs. the relative plate bluntness at $H / h=2, \mathrm{M}=5(1)$ and $8(2)$

As the blunting radius increases to $r=0.75 \mathrm{~mm}$ the signs of the mixing-layer turbulization appear. At the same time, the inclination angles of the separation shock and the separation zone boundary increase (up to $15.4^{\circ}$ and $10^{\circ}$, respectively).

Behind the channel throat, for example, in section 2, a variation of the free flow Mach number in the investigated range influences the degree of the heattransfer reduction lightly (the ratio $\mathrm{St}_{m} / \mathrm{St}_{m s}$, Fig. 18b). On the contrary, in front of the channel throat the blunting influence on the heat transfer increases with the increase of Mach number. The same is observed at the flow over a single fin and at the 2D flow. Thus, the Mach number affects the heat transfer

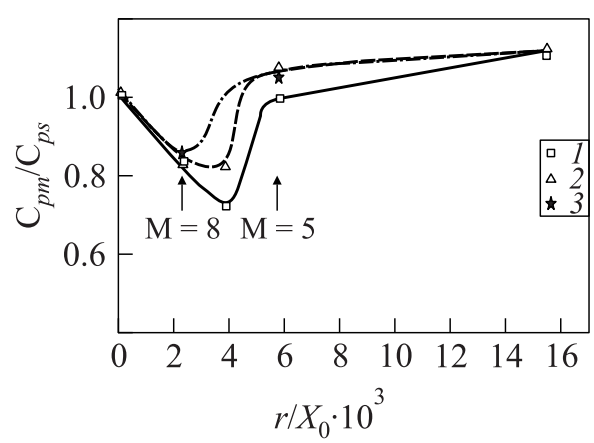

(a)

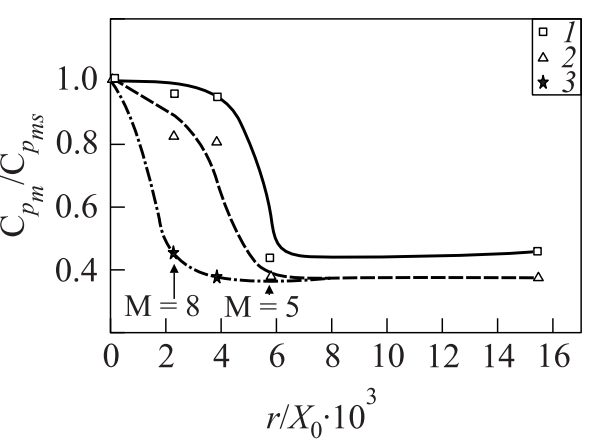

(b)

Figure 19 Maximum values of pressure coefficient in cross sections $1(a)$ and $2(b)$ vs. the relative plate bluntness at $\mathrm{M}=5(1), 6(2)$, and $8(3) ; H / h=4$. The arrows denote the flow transformation 
in the interaction zone differently, depending on the flow type. This problem deserves further investigation. Figure 19 demonstrates the blunting influence on the maximum pressure in sections 1 and 2 at $M=8$. It can be seen that expelling of the separation shocks terminates the monotony of the pressure dependences on the bluntness radius: after shocks expelling, the pressure drop in front of the channel throat (in section 1) is changed into its increase, and behind the throat (in section 2), the pressure drop stops.

Comparing the results of experiments at different Mach numbers, it should be noted that the Reynolds number is varied simultaneously with the Mach number. It may be supposed that Mach number variation is more important for the flow transformation than the Reynolds number variation in the studied range $\left(\operatorname{Re}_{\infty L}=7 \cdot 10^{6}-27 \cdot 10^{6}\right)$. But this problem needs farther investigation.

\section{CONCLUDING REMARKS}

The surface flow and the heat transfer on the sharp and blunted plates in the presence of a fin or a fin pair, generating crossing shocks, are investigated at Mach numbers $\mathrm{M}=5,6$, and 8 and Reynolds numbers up to $\operatorname{Re}_{\infty L}=27 \cdot 10^{6}$. It is shown that the entropy layer generated by the small blunting of the plate leading edge changes significantly the heat transfer, the pressure, and the skin friction in the region of the shock-wave/boundary-layer interaction, and at some conditions, it can cause a transformation of the flow structure. In particular, the following was established.

In the flow around a single fin, the separation zone is formed with the reattachment line situated at the fin. Near the fin leading edge (in the initial region), the values of the heat transfer and pressure coefficients on the reattachment line increase gradually downstream the fin leading edge. Maximum values of $\mathrm{St}_{m}$ and $C_{p_{m}}$ are achieved only at a significant distance from the fin leading edge (in the region of quasi-conical flow). The length of the initial region increases at the blunting increase.

The maximum values of the heat transfer and pressure coefficients $\left(\mathrm{St}_{m}\right.$ and $C_{p_{m}}$ ) near the single fin decrease as the blunting radius of the plate leading edge increases. Simultaneously the region of amplified heat transfer and increased pressure expands. At a certain value of the relative blunting radius $r / X_{0}$, significant changing of $\mathrm{St}_{m}$ and $C_{p_{m}}$ terminates. The described influence of the plate blunting on the gas flow is observed at all the investigated fin angles. It amplifies at the increase of Mach number.

Results of the numerical calculation of the Stanton number distribution near the single fin, implemented using the $q-\omega$ turbulence model, comply with the experimental data satisfactorily. 
In the case of a fin pair, generating crossing shocks, the heat transfer and the pressure behind the intersecting shocks increase significantly in comparison with the case of a single fin. Maximum heat transfer and pressure coefficients behind the crossing shocks increase at the increase of the contraction ratio of the channel between the fins.

At a high channel contraction ratio, the separation shocks are expelled from the channel towards the undisturbed flow when the plate blunting radius exceeds a certain critical value. At that, one vast separation zone is formed instead of two narrow separation zones situated near the fins. It begins at a large distance in front of the fins and terminates at the channel throat. The flow transformation changes the heat transfer and pressure distributions both in front of the fins and between them. The critical blunting value decreases at Mach number increase.

As in the case of a singe fin, the increase of the plate blunting radius within a certain range (up to $r / X_{0} \approx 0.015$ ) reduces the heat transfer and the pressure in the interaction region significantly, if the regular flow is kept (i. e., without expelling the separation shocks).

\section{ACKNOWLEDGMENTS}

The research was supported by International Science and Technology Center (ISTC) under Grant \#3872 and Russian Foundation for Basic Research (RFBR) under Grants No. 08-01-00449 and 11-01-00657.

\section{REFERENCES}

1. Miller, D. S., R. Hijman, E. Redeker, W.C. Janssen, and C. R. Mullen. 1962. A study of shock impingement on boundary layer at Mach 16. Heat Transfer and Fluid Mechanics Institute. Stanford, CA: Univ. Press. 255-78.

2. Borovoy, V. Ya., and E. V. Sevastyanova. 1973. Gas flow and heat exchange in the region of interaction of a laminar boundary layer with the shock wave near a halfwing, installed on the plate. Uchenye Zapiski TsAGI 4(2):54-63. [In Russian.]

3. Zheltovodov, A.A. 1979. Physical peculiarities and some features of twodimensional and three-dimensional flows with separation at supersonic velocities. Izv. Russ. Acad. Nauk, Mekhanika Zhidkosti Gaza 3:42-50. [In Russian.]

4. Knight, D. D., C. C. Horstman, B. Shapey, and S. Bogdonov. 1987. Structure of supersonic turbulent flow past a sharp fin. AIAA J. 25(10):1331-37.

5. Kussoy, M. I., K. C. Horstman, and C. C. Horstman. 1993. Hypersonic crossing shock-wave/turbulent-boundary-layer interactions. AIAA J. 31(12):2197-203. 
6. Garrison, T. J., G.S. Settles, N. Narayanswami, and D. D. Knight. 1993. Structure of crossing-shock-wave/turbulent-boundary-layer interactions. AIAA J. 31(12):2204-11.

7. Knight, D., H. Yan, A. G. Panaras, and A. Zheltovodov. 2003. Advances in CFD prediction of shock wave turbulent boundary layer interactions. Prog. Aerospace Sci. 39(2-3):121-84.

8. Schulein, E. 2006. Skin-friction and heat flux measurements in shock/boundarylayer interaction flows. AIAA J. 44(8):1732-41.

9. Borovoy, V. Ya., I. V. Egorov, A. S. Skuratov, and I. V. Struminskaya. 2005. Interference of an oblique shock with the boundary and high entropy layers of the flat plate. Izv. Russ. Acad. Nauk, Mekhanika Zhidkosti Gaza 40(6):89-108.

10. Borovoy, V. Ya., A. S. Skuratov, and I. V. Struminskaya. 2008. On the existence of a threshold value of the plate bluntness in the interference of an oblique shock with boundary and entropy layers. Izv. Russ. Acad. Nauk, Mekhanika Zhidkosti Gaza $43(3): 41-52$.

11. Borovoy, V. Ya., I. V. Egorov, A. S. Skuratov, and I. V. Struminskaya. 2011. Twodimensional interaction of the oblique shock wave with the boundary and highentropy layers of the blunt plate. AIAA Paper No. 2011-731.

12. Borovoy, V., V. Mosharov, V. Radchenko, and A. Noev. 2012. Laminar-turbulent flow around a wedge placed on sharp and blunted plates. In: Progress in flight physics. Eds. Ph. Reijasse, D. D. Khight, M. S. Ivanov, and I. I. Lipatov. Advances in aerospace sciences EUCASS book ser. 3:307-20.

13. Borovoy, V., V. Mosharov, A. Noev, and V. Radchenko. 2012. Temperature sensitive paint application for investigation of boundary layer transition in short-duration wind tunnels. In: Progress in flight physics. Eds. Ph. Reijasse, D. D. Khight, M. S. Ivanov, and I. I. Lipatov. Advances in aerospace sciences EUCASS book ser. 3:15-24.

14. Huang, F. G., and T. J. Coakley. 1993. Turbulence modeling for high speed flows. AIAA Paper No. 92-0436. 